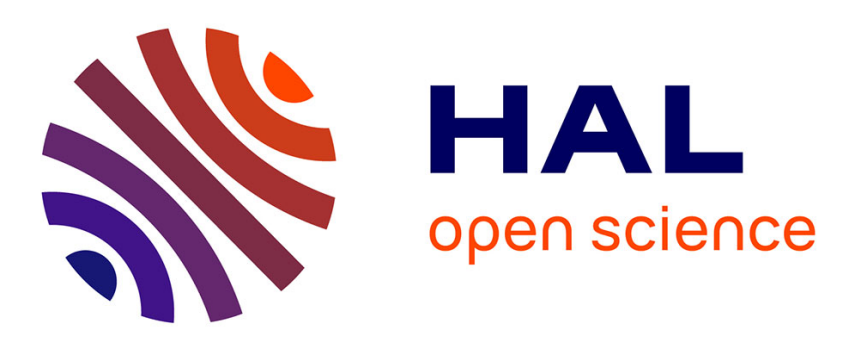

\title{
Improving Rural Emergency Services with Cognitive Radio Networks in Sub-Saharan Africa
}

Dramane Ouattara, Francine Krief, Mohamed Aymen Chalouf, Tegawendé F. Bissyandé

\section{- To cite this version:}

Dramane Ouattara, Francine Krief, Mohamed Aymen Chalouf, Tegawendé F. Bissyandé. Improving Rural Emergency Services with Cognitive Radio Networks in Sub-Saharan Africa. AFRICOMM 2013, Fifth International IEEE EAI Conference on e-Infrastructure and e-Services for Developing Countries, Nov 2013, Blantyre, Malawi. 10 p. hal-00998859

\section{HAL Id: hal-00998859 \\ https://hal.science/hal-00998859}

Submitted on 2 Jun 2014

HAL is a multi-disciplinary open access archive for the deposit and dissemination of scientific research documents, whether they are published or not. The documents may come from teaching and research institutions in France or abroad, or from public or private research centers.
L'archive ouverte pluridisciplinaire HAL, est destinée au dépôt et à la diffusion de documents scientifiques de niveau recherche, publiés ou non, émanant des établissements d'enseignement et de recherche français ou étrangers, des laboratoires publics ou privés. 


\title{
Improving Rural Emergency Services with Cognitive Radio Networks in Sub-Saharan Africa
}

\author{
Dramane Ouattara ${ }^{1}$, Francine Krief ${ }^{1}$, Mohamed Aymen Chalouf ${ }^{2}$, and \\ Tegawendé F. Bissyandé ${ }^{3}$ \\ 1 University of Bordeaux, LaBRI, 351 cours de la Libération \\ 33405 Talence Cedex, France \\ dramane.ouattara@labri.fr, francine.krief@labri.fr \\ 2 University of Rennes 1, IRISA, IUT of Lannion, Rue Edouard Branly \\ 22300 Lannion, France, mohamed-aymen.chalouf@irisa.fr \\ 3 University of Luxembourg, Campus Limpertsberg, av. de la Faïencerie \\ 162 A, L-1511 Luxembourg, tegawende.bissayande@uni.lu
}

\begin{abstract}
In this paper, we propose a new approach based on Cognitive Radio technology to address the challenges for ensuring connectivity in remote areas of Africa. Indeed, the current network coverage is concentrated around the cities with high density of population. Through the deployment of Cognitive Radio, emergency services in rural areas will benefit from low cost access networks. Cognitive Radio will be used to manage the selection/switching across different frequency UHF/VHF bands or TV White Spaces.
\end{abstract}

Key words: Cognitive Radio, TV White Spaces, Rural areas, Remote zone connectivity.

\section{Introduction}

The use of mobile technologies and networks in Africa is growing rapidly and services are increasingly diversified. The quality of the available networks differs from one geographical area to another. In many areas, the networks deliver a poor connectivity, while some areas have no connectivity possibility. These deficiencies are related to the operators policies and the economic benefits they should derive from their investment. However, Internet or networks access could considerably improve the inhabitants social condition in remote areas. Providing low cost Internet access anywhere through Cognitive Radio Networks (CRNs) is the main objective of this contribution. In this paper, we introduce the Cognitive Radio technology, with a more detailed description of its main modules in section 2. The main advantages of this technology in African context are presented in section 3 through the services that it could offer. We give an overview on the related work, referring to some work addressing the use of cognitive radio in African context in section 4 . The deployment plan that we propose is considered in section 5, an experimentation idea and results are studied in 6. Conclusion and future work are presented in section 7 . 


\section{The cognitive radio networks}

\subsection{Definition and principle of cognitive radio networks}

Cognitive Radio [1] is a paradigm for wireless networks where a node is able to automatically modify its transmitting parameters in order to communicate efficiently, while avoiding interferences with other users, the Primary Users $\left(\mathrm{PU}^{1}\right)$. This self-configuration and self-adaptation of parameters is based on a set of modules and several factors in the internal or the external environment of the radio such as radio frequency, user behaviour and the network state.

\subsection{Cognitive radio modules}

Figure 1 summarizes the cognitive radio modules and details of its functions are given below.
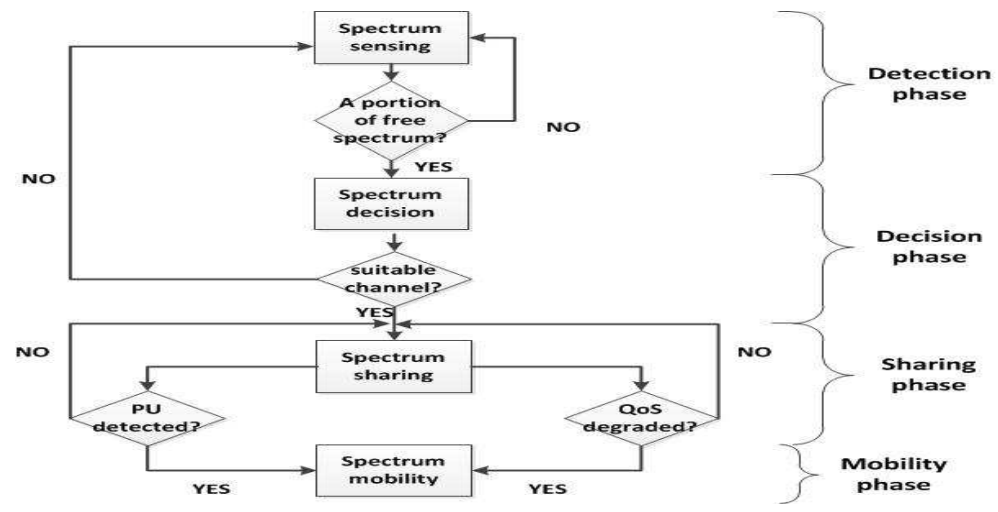

Fig. 1: Operating diagram of a cognitive radio node

Spectrum sensing: The spectrum sensing is defined as the ability to measure, examine, learn and be aware of the parameters related to the characteristics of the radio channel. This module measures the availability of spectrum, the signal strength, the interferences and noise, scans operating environment of the radio, estimates the needs of users and applications, checks the availability of networks and nodes, learn about the local policies and other operators restrictions.

Spectrum decision: Decision-making is based on the appropriate communication channel choice, justifying the quality of service required for the data or the collected information transmission.

Spectrum sharing: Channel sharing has to comply with the requirement of synchronized access to the detected free-bands portions. This scheduling is

\footnotetext{
${ }^{1}$ Users that have the band-use license, the TV users.
} 
done between the secondary ${ }^{2}$ users on the one hand, and between these users and the primary users on the other hand.

Spectrum mobility: Spectrum mobility reflects the fact that each transceiver, must be able to change frequency band if the initial band becomes busy. Moving to a new frequency band could happen also when the initial band fails to provide the desired quality of service to service's applications.

\subsection{The standard for cognitive radio: IEEE 802.22}

The Federal Communications Commission $\left(\mathrm{FCC}^{3}\right)$ established the TV white spaces rules by which unlicensed devices, in our case, the cognitive radio devices, can make use of specific TV channels in the Very High Frequency (VHF) and Ultra High Frequency (UHF) bands. The table below summarises these frequency bands.

\begin{tabular}{|l|l|l|c|c|c|}
\hline Channel & Spectrum & Bands & Channel & Spectrum & Bands \\
\hline $2,3,4$ & $54-72 \mathrm{MHz}$ & VHF Low & $7-13$ & $174-216 \mathrm{MHz}$ & VHF High \\
\hline 5,6 & $76-88 \mathrm{MHz}$ & VHF Low & $14-51$ & $470-698 \mathrm{MHz}$ & UHF \\
\hline
\end{tabular}

The use of cognitive radio approach in rural areas in Africa is based on this principle of TV white spaces, even if the constraints and objectives are different from European and American continents. Indeed, the scarcity of spectrum resources in Europe and America led to the exploration of this new alternative. In Africa, it will provide technology services in regions with limited economic resources, often remote areas at a very insignificant cost or even free. The use of TV White Spaces in Africa to ensure a connection is a topic increasingly studied and therefore, has recently been the subject of the TV white spaces Africa Forum in Dakar (Senegal) with partners such as Google and Microsoft[2]. The 802.22 working group is developing standards for wireless regional network based on TV white spaces usage. Here are some specifications of the standard on cognitive radio ${ }^{4}$ :

\begin{tabular}{|l|l|}
\hline Standards & Description \\
\hline IEEE 802.22.1 & Interferences avoidance, primary TV users protection \\
\hline IEEE 802.22.2 & Practice for the systems deployment and installation \\
\hline
\end{tabular}

This table shows the work in progress and all the interest in the use of TV bands to provide internet services.

\section{Services that could be offered}

Given the ability of the Cognitive Radio Networks to realize opportunistic communications, this technology could provide a set of services in rural zones, among these are:

\footnotetext{
${ }^{2}$ Cognitive radio users, who do not have any band-use license, such as emergency services users in our context.

${ }^{3}$ http://www.fcc.gov/

${ }^{4}$ IEEE 802.22-2011 Standard for Wireless Regional Area Networks, July 27th 2011.
} 


\subsection{Chronic disease patient monitoring : e.g diabetic patient}

Chronic diseases such as diabetes are reaching an increasingly large proportion of the rural population. Patients in rural areas with appropriate monitoring, combine with timely hospital visits should save lives. In fact, diabetic patient fitted with glucose sensors connected to a smart-phone as a relay node to the internet allows to remotely inform the caregivers on abnormal high levels of sugar in the blood. This could avoid many painful movement of the patients from rural areas to the city (Hospital). The principle remains the same for other types of chronic conditions such as cardiovascular, cancer and respiratory diseases.

\subsection{Hospital services automation : e.g for epidemic disease prevention}

To accelerate treatment and diagnosis, health services in Africa should be automated. It begins with the electronic record of the patient's medical history and allows the anywhere medical records access even for people living in rural areas. The patients often helped by nurses could access, modify and control remotely their medical information or send a message on his health state to the doctors. The statistics from this automation could be helpful for early detection of health risks such as epidemics.

\subsection{Emergency alerts : e.g Bushfire and accident alerts}

Bushfire are often disastrous for people in rural areas and often, these people do not have the ability to call for help due to the lack of communication infrastructure. It is the same for accident occurring in very remote areas where the victims have no access networks to call the firemen. The emergency networks seem enough efficient for saving lives in similar situation with rescue arrival on time.

\subsection{Internet for children}

In addition to emergency centers that could benefit from Internet, the primary schools should be connected and the children in remote villages could very soon become familiar with computers and social networks. This gives them an opening to the world, an opportunity to interact with other children, thus contributing to the reduction of social and technological gap.

\subsection{Improving government services : e.g births registration}

Child births in villages are often not reported because of the distance to reach an administration office. This raises the problem of persons with no administrative paper for example in Côte d'Ivoire remote areas. The on-line registration of births through cognitive radio could significantly reduce the problem of undocumented persons. At the same time, several administrative services may be offered by the Internet access in villages. 


\section{Related Works}

In developed countries, the growing number of wireless devices and the increased spectrum occupancy have resulted to the spectrum scarcity. Cognitive Radio is considered in Europe and USA as the new wireless communication paradigm that could address the potential spectrum exhaustion problem and should be proposed for future wireless communication devices. In the African context, this technology could mostly serve as knowledge sharing and social development tool. Cognitive Radio Networks are therefore a promising field for social networks deployment in Africa and the domain has an increasingly interest for researchers. Thus, in [3], the White space opportunity has been studied. The authors performed measurements that indicate the existence of substantial TV White Spaces available in both rural and urban areas. This work is an interesting introduction and opens up practical deployment studies which remain unexplored. Implementation of OpenBTS in rural Zambia has been studied in [4]. This work focused on providing telecommunication system such as mobile communications in rural villages. Even if this study addresses the low cost communication issue, the solution is obviously valid and valuable for the only villages with GSM networks infrastructure. However, the African countries reality proves that the majority of villages are not covered by the existing standard networks. In fact, the economic profitability in terms of return on investment is not guaranteed for telecommunications operators. The use of Cognitive Radio technology becomes therefore necessary with its bearable costs because of the existence of TV bands infrastructure in rural areas.

\section{Cognitive Radio deployment process in remote areas}

Depending on the isotropic radiated power, the cognitive radio base station could connect users terminals located as far as $100 \mathrm{~km}[5]$ as described in Figure 2. A good base stations planning could provide a network to cover two distant areas (villages) of about 100 and greatly reduce the cost of network infrastructure. The financing by governments and the acquisition of such infrastructure and its deployment will aim to improve public services in Africa remote rural areas.

\subsection{Cognitive radio networks planning scenario}

Figure 2 describes the deployment scenario with a set of cities (c), villages (v) with distances estimation and the corresponding coverage plan. The cognitive radio antennas at the city allow the switching between our cognitive radio network and the existing operators networks. Also, the cognitive radio antennas set near a city, due to their sensing capability will help avoiding interferences that may be generated by the broadcasting signal of our cognitive radio networks. Relay nodes (R1) are provided to repeat the signal when the distance between two access points or base stations is greater than $100 \mathrm{~km}$. Thus, it can be seen that the fundamental interest of this proposal lies in its ability to cover a large area and long distance. It should be noted that the TV band could be used only in areas lacking the standard network coverage. A proper planning 


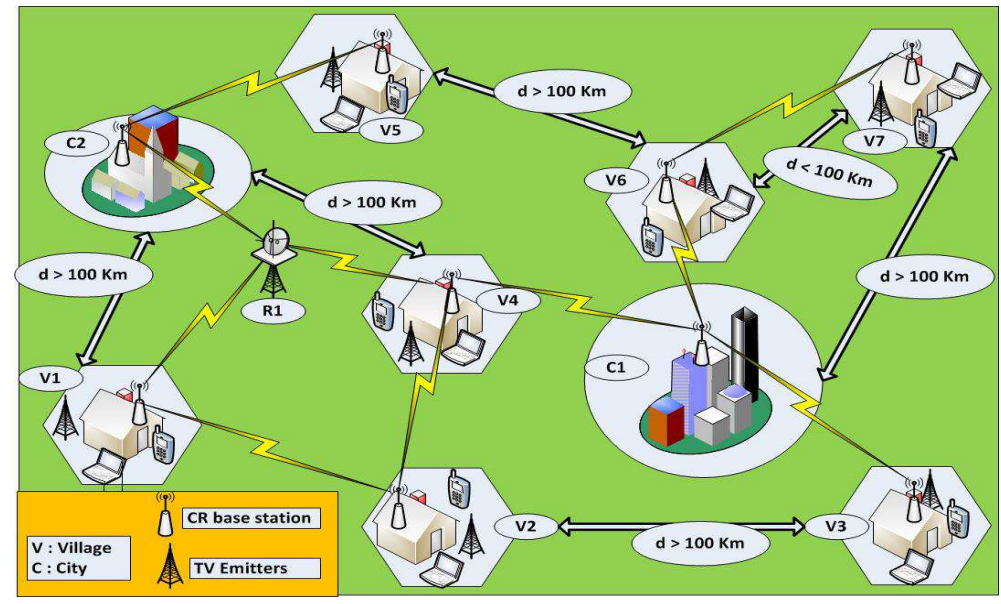

Fig. 2: Planned Cognitive Radio Networks

and deployment will achieve a very high scope for possible internet access in the most remote locations. The cognitive radio is therefore an extension of network coverage anywhere and any-time even if its access must be controlled and limited to emergency services, public safety services or public services to avoid disorder in its usage. Based on a strong existing infrastructure such as TV bands (TV White Spaces), the solution we propose provides a stable network connection and facilitates its management and control. However, a number of challenges remain to make the effective deployment of this technology in rural areas in Africa.

\subsection{Challenges}

The challenges in this context concern the spectrum sensing for better detection of free TV frequencies and channel sharing. A good algorithm for free bands detection will reduce interferences and a good signalling protocol will prevent collisions.

Interferences avoidance with TV users: The deployment of cognitive radio networks creates a new type of opportunistic users whose major constraint remains achieving transmissions or communications without interfering with the TV users. Figure 3 shows the general principle used by the cognitive radio Medium Access Control (MAC) protocols for collisions mitigation. This figure illustrates the different steps performed by a cognitive radio node before the frequency band access. There is a sensing period (scan), a period of reconfiguration and synchronization before any transmissions on the detected free channels (useful time). Occupied primary channels denote in this case, the occupation of the frequency band by TV users and useful time are periods that can be exploited by the cognitive radio users for their transmissions.

Network performance enhancement: A better network performance in terms of throughput and available bandwidth is an important interest in ensuring quality of service to users. Performance is thus linked to the availability 


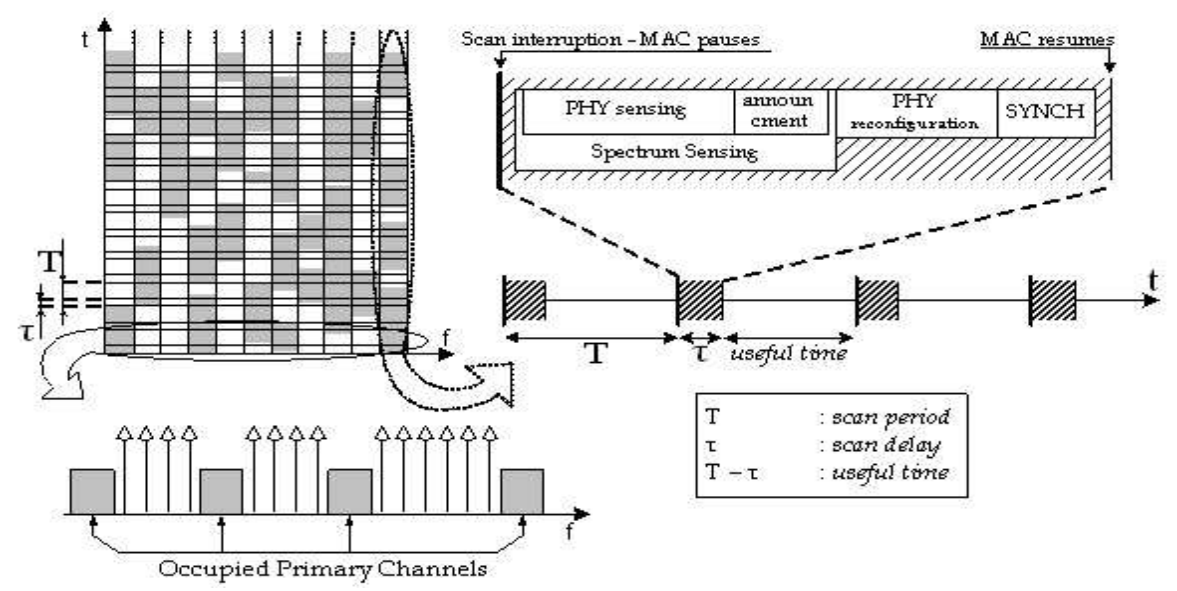

Fig. 3: Spectrum analysis for the cognitive users access[6]

of channels and the quality of these free bands. The work proposed in [7] on modelling the behaviour of primary users (TV users in this context) shows how to improve the process of detection of free bands and reduce latencies due to the frequency hopping induced by a primary user probable appearance. In addition, others works [8] shown the capacity of cognitive radio technology to provide high throughput and good quality of service for users.

Energy for powering equipments and maintenance: The access to a source of energy to power the equipment to be installed is a real challenge for the deployment of cognitive radio networks in rural areas. In fact, rural areas in Africa are devoid of electric networks, however, there are alternatives such as solar energy increasingly used. Generators are also used as power source for electronic equipment and research continues to provide solutions to electricity problems in areas with limited resources (rural area) [9]. The energy problem can not therefore constitute an obstacle to the deployment of cognitive radio technology in remote areas.

Engineering and deployment: For better reduction of costs, a proper study should be conducted, that enables efficient deployment of network infrastructure. Thus, must be taken into account the best locations for cognitive radio base stations and relay nodes in order to minimize the infrastructure's cost while maximizing the network coverage area.

\section{Experiments and results}

In our research work on cognitive radio networks, we perform experiments and tests on a platform acquired under the $\mathrm{ANR}^{5}$-LICORNE project and a platform offered by the CREW project.

\footnotetext{
${ }^{5}$ The French National Research Agency - ANR (Agence Nationale de la Recherche en France).
} 


\subsection{The cognitive radio platform LICORNE}

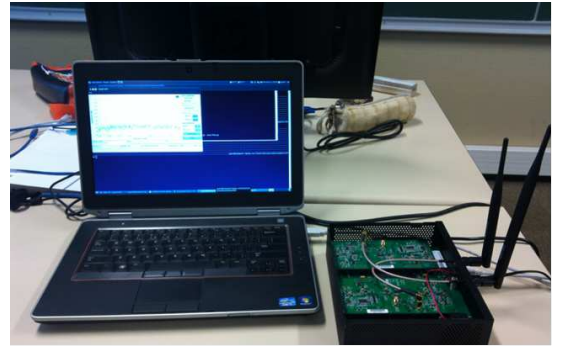

Fig. 4: A cognitive radio node

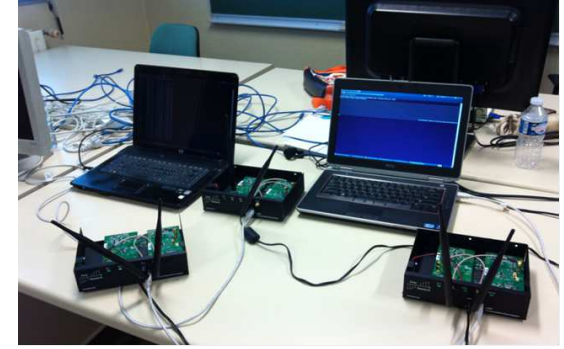

Fig. 5: Three nodes connected

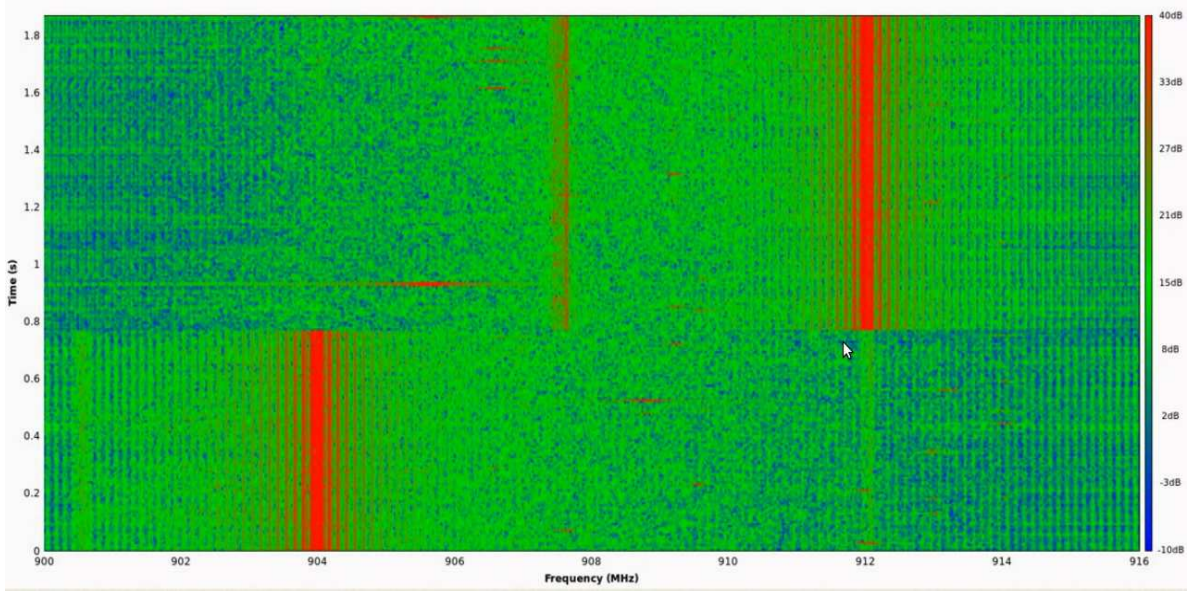

Fig. 6: Frequency hopping for interferences avoidance

This platform is composed of five nodes and each node is composed of a USRP-1 box, two daughter-boards for transmission/reception (Tx/Rx) operating in GSM (RX/TX 900) and in WiFi (RX/TX 2400) accompanied with the corresponding antennas. A laptop with GNU software is interconnected to the USRP box as presented in Figure 4. This platform allowed us to do an experiment of transmission / reception on WiFi and GSM bands and to test the frequency hopping with the setup shown in Figure 5.

To test the frequency hopping, we generate a disruptive AWGN (Adds White Gaussian Noise) signal to create interferences on the transmission channel. The signal thus generated is seen as a transmission of a primary node. This then triggers the process of frequency hopping to avoid interferences as seen in Figure 6. This experiment in GSM and $\mathrm{WiFi}$ bands remains valid for mitigating 
interferences to TV band users with cognitive radio networks deployed in rural areas. In addition, the transmission power adaptation model proposed in [10] and tested through the LICORNE platform, represents another strategy for mitigating interferences in cognitive radio networks and therefore for TV white spaces.

\subsection{The CREW project platform}

The CREW platform [11] facilitates experimentally-driven research on spectrum sensing and sharing in licensed and unlicensed bands (TV bands). It offers testbed capabilities to TV frequency bands experimenters. It Combined indoor and outdoor installation deployed in the city of Logatec at Slovenia. The test-bed remote access portal ${ }^{6}$ allows to show node status, choose particular cluster for performing an experiment remotely as shown in Figure 7.

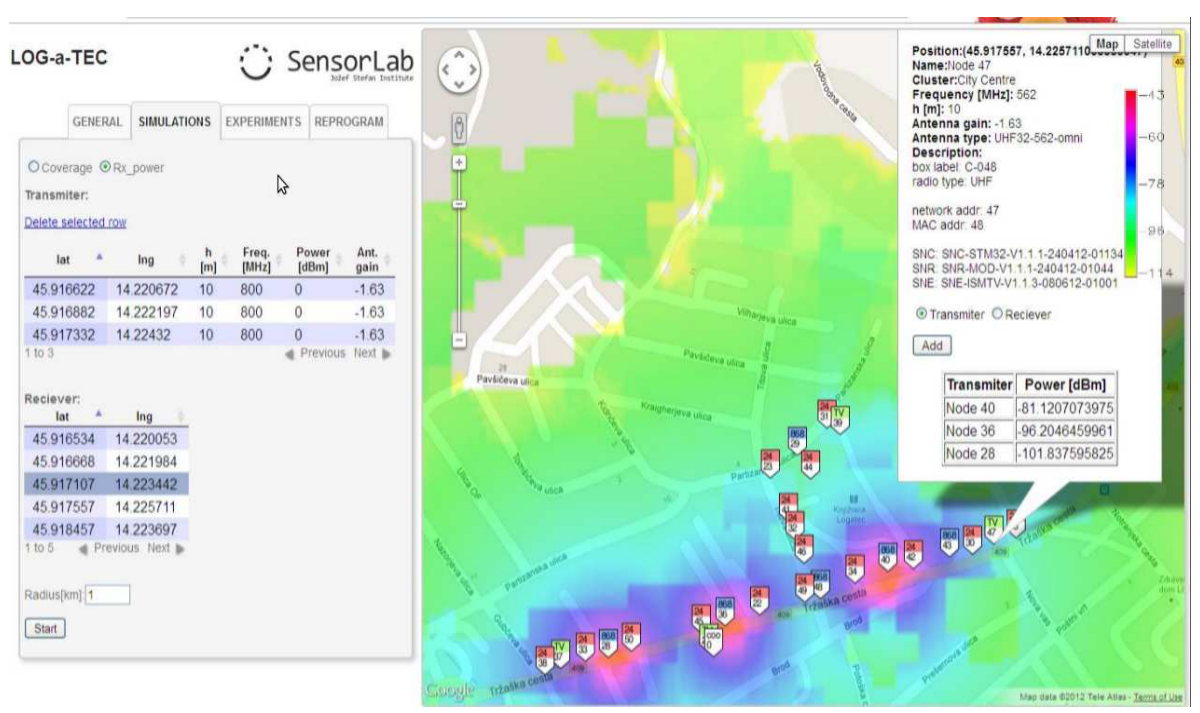

Fig. 7: Platform for remote test on the TV bands

We worked on this platform during the CREW training days ${ }^{7}$ and made tests remotely to validate the TV frequency bands potential in terms of data transmissions and interferences awareness as seen in Figure 7.

The transmitters radio coverage calculation and visualisation is expressed through the Figure 7. The different colors (green, blue, red) and the characteristic of energy detected from one zone to another one, allows to confirm the presence or the absence of a transmission based on the measured power. Also, this platform (CREW) allows for various tests such as the multi-hopping scenario that remains a very important aspect for transmissions reaching remote areas.

\footnotetext{
${ }^{6}$ www.log-a-tec.eu

7 CREW Training Days is the day which took place on February 2013 at Brussels
} 


\section{Conclusion}

In this work, we have shown that cognitive radio is a real opportunity for network coverage in rural areas through the use of TV band. We have proposed a scenario for the deployment of this technology, noting its expansion capacity over long distances to ensure network access in remote areas. The set of services (section 3) that can be offered through the cognitive radio networks demonstrates its importance in African context. This next generation network is promising to improve life of many people living in remote and often inaccessible regions in Africa. In the future, we will propose a communication architecture based on cognitive radio networks to improve health services in rural areas. Health centers to be interconnected, medical applications to develop and install, patient to be remotely monitored are some challenges for our future proposal in the context of rural areas in Africa.

\section{Acknowledgment}

This work is partially supported by the LICoRNe project, funded in part by the National Agency for Research in France ANR (Agence Nationale de la Recherche).

\section{References}

1. J. Palicot. : Cognitive Radio: An Enabling Technology for the Green Radio Communications Concept, Leipzig, Germany - June 21 - 24, 2009.

2. Forum website: https://sites.google.com/site/tvwsafrica2013/

3. Moshe T. Masonta, David J. and Mjumo Mzyece : The White Space Opp. in Southern Africa : Measurement with Meraka C.R. Platform : AFRICOMM 2011.

4. Jacqueline M. and Gertjan van S. : Open BTS, a GSM experiment in rural Zambia : 4th Int. Conf. ICST, AFRICOMM 2012

5. Alexander M. Wyglinski,Maziar Nekovee,Thomas Hou : Cognitive Radio Communications and Networks: Principles and Practice,Academic Press, 13 nov. 2009.

6. Athanassios V., Adamis and Philip Constantinou : Intermittent DCF: a MAC protocol for Cognitive Radios in Overlay Networks; Cognitive Radio Systems; book edited by Wei Wang, november 2009.

7. Dramane Ouattara; Francine Krief; Mohamed Chalouf; Omessaad Hamdi: Spectrum Sensing Improvement in Cognitive Radio Networks for Real-Time Patients Monitoring; Wireless Mobile Communication and Healthcare; Third International Conference, MOBIHEALTH 2012.

8. Umamaheswari, A.; Subashini, V.; Subhapriya, P., Survey on performance, reliability and future proposal of Cognitive Radio under wireless computing, Computing Communication (ICCCNT), 2012 Third Int. Conf. July 2012.

9. Gado, Abla; El-Zeftawy, Atef, Design and economy of renewable energy sources to supply isolated loads at rural and remote areas of Egypt, Electricity Distribution, CIRED, 20th International Conference and Exhibition June 2009

10. Dramane Ouattara; Minh T. Quach; Francine Krief; Mohamed A. Chalouf; Hicham Khalife: Mitigating the hospital area communication's interference using cognitive radio networks; IEEE Healthcom, International conf. on E-health networking, application and services; October 2013.

11. http://www.crew-project.eu/ 